\title{
Comparison Between Extracorporeal Shock Wave Therapy and Intra-articular Hyaluronic Acid Injections in the Treatment of First Carpometacarpal Joint Osteoarthritis
}

\author{
Francesco Ioppolo, $\mathrm{MD}^{1}$, Fabiana Saracino, $\mathrm{MD}^{1}$, Rosaria Sabrina Rizzo, $\mathrm{MD}, \mathrm{PhD}^{1}$, \\ Giampaolo Monacelli, $\mathrm{MD}^{2}$, Danilo Lanni, $\mathrm{MD}^{1}$, Luca Di Sante, $\mathrm{MD}, \mathrm{PhD}^{1}$, \\ Angelo Cacchio, $\mathrm{MD}, \mathrm{PhD}^{3}$, Valter Santilli, $\mathrm{MD}, \mathrm{PhD}^{1}$, Teresa Venditto, $\mathrm{MD}^{1}$ \\ ${ }^{1}$ Department of Physical Medicine and Rehabilitation, Sapienza University of Rome, Rome; \\ ${ }^{2}$ Emergency Department of Plastic Surgery and Hand Surgery, Sapienza University of Rome, Rome; \\ ${ }^{3}$ Department of Life, Health and Environmental Sciences, School of Medicine, University of L'Aquila, L'Aquila, Italy
}

\begin{abstract}
Objective To compare extracorporeal shockwave therapy (ESWT) with hyaluronic acid (HA) intra-articular injections in terms of pain relief, improvement in hand function, and strength in subjects with first carpometacarpal (CMC) joint osteoarthritis. Methods Fifty-eight patients received either focused ESWT or HA injection once a week for 3 consecutive weeks. In the ESWT group, 2,400 consecutive pulses were performed during each treatment session using a frequency of $4 \mathrm{~Hz}$ and an an energy flux density of $0.09 \mathrm{~mJ} / \mathrm{mm}^{2}$. The HA group underwent one cycle of three injections of $0.5 \mathrm{~cm}^{3} \mathrm{HA}$. The main outcome measures were pain and hand function as measured by the visual analogue scale (VAS) and Duruoz Hand Index (DHI), respectively. The secondary outcomes were grip and pinch strength. Each assessment was performed at baseline, at the end of treatment, and at 3- and 6-month follow-up visits.

Results According to VAS and DHI scores, a significant change in test performance was observed over time in both groups $(\mathrm{p}<0.001)$, with a greater average improvement in painful symptomatology at the 6-month follow-up in the ESWT group. A significant improvement in strength was observed in both groups, but the ESWT group showed better results on the pinch test starting immediately at the end of treatment.

Conclusion The use of ESWT in patients with first CMC joint osteoarthritis leads to a reduction in pain, an improvement in pinch test performance that persists for at least 6 months, and a decrease in hand disability up to the 6-month follow-up visit.
\end{abstract}

Keywords Extracorporeal shock wave therapy, Hyaluronic acid, Thumb, Osteoarthritis

Received July 18, 2016; Accepted September 13, 2016

Corresponding author: Teresa Venditto

Department of Physical Medicine and Rehabilitation, Sapienza University of Rome, Piazzale Aldo Moro 5, Rome 00185, Italy. Tel: +39-0649975912, Fax: +39-0649914547, E-mail: teresa.venditto@hotmail.it.

ORCID: Francesco Ioppolo (http://orcid.org/0000-0003-1586-9043); Fabiana Saracino (http://orcid.org/0000-0001-5770-9262); Rosaria Sabrina Rizzo (http://orcid.org/0000-0001-8439-9853); Giampaolo Monacelli (http://orcid.org/0000-0002-8517-5189); Danilo Lanni (http://orcid.org/0000-00034320-8656); Luca Di Sante (http://orcid.org/0000-0002-2200-0445); Angelo Cacchio (http://orcid.org/0000-0001-8850-7938); Valter Santilli (http:// orcid.org/0000-0002-2547-6801); Teresa Venditto (http://orcid.org/0000-0003-4638-0734).

(a) This is an open-access article distributed under the terms of the Creative Commons Attribution Non-Commercial License (http://creativecommons.org/ licenses/by-nc/4.0) which permits unrestricted noncommercial use, distribution, and reproduction in any medium, provided the original work is properly cited. Copyright $\odot 2018$ by Korean Academy of Rehabilitation Medicine 


\section{INTRODUCTION}

Osteoarthritis (OA) is a chronic degenerative disease with a significant impact on a patient's quality of life [1]. This pathology can involve different joints of the body but, when limited to the hand, it is frequently localized to the first carpometacarpal (CMC) joint. First CMC joint OA manifests with pain, swelling, stiffness, and deformity at the base of the thumb. If the condition is left untreated, patients may develop a severe adduction contraction of the thumb with a subluxation of the CMC joint, which can lead to impairments in hand function with a gradual loss of dexterity and a deterioration of intrinsic muscle strength [2].

Conservative treatment is the usual first step in the management of first CMC joint OA. Commonly used treatments include rest, anti-inflammatory drugs, occupational therapy, night splinting, and intra-articular (IA) steroid or hyaluronic acid (HA) injections [3-5].

Intra-articular corticosteroid use is relatively safe, but side effects may occur after repeated injections, such as local infection due to immunological suppression, local tissue atrophy, tendon and ligament weakening, and peri-articular soft tissue calcification [6]. On the other hand, intra-articular HA injections result in viscosupplementation, reestablishing the hand's elastic properties, such as cushioning, lubrication, and elasticity, restoring joint rheology, and stimulating anti-inflammatory effects [7]. In a recent systematic review, Spaans et al. [8] observed the effectiveness of both treatments in terms of pain relief, and found a much more long-lasting effect with hyaluronate injection. Moreover, in a randomized controlled study, Monfort et al. [9] assessed the efficacy and safety of ultrasound-guided intra-articular injections of low-molecular-weight HA into the first CMC joint in comparison with corticoid injections. Both hyaluronic acid and betamethasone were effective and well-tolerated, but HA showed a long-lasting effect, especially in patients with severe symptoms.

However, choosing a specific treatment is still controversial because of limited evidence concerning which procedure provides the best results with minimal complications. Therefore, new therapeutic strategies should be introduced in the management of first CMC joint OA.

Extracorporeal shockwave therapy (ESWT) has proven to be effective in treating musculoskeletal disorders [10-
13] owing to its angiogenic analgesic and anti-inflammatory effects $[14,15]$.

To date, few studies have assessed the usefulness of ESWT in treating OA. Some of them evaluated the effectiveness of the treatment on animals $[16,17]$. Other studies were carried out on human articular cartilage obtained from patients suffering from primary knee OA [18], and only one clinical trial evaluated the efficacy of ESWT in patients suffering from knee osteoarthritis [19].

The purpose of the present study was to compare the effectiveness of low-energy ESWT versus HA intra-articular injections in terms of pain relief and improvements in hand function and strength in subjects with first CMC joint OA.

\section{MATERIALS AND METHODS}

\section{Study design and participants}

This study was conducted to assess the clinical role of ESWT in the management of first CMC joint OA, and to compare its efficacy with that of intra-articular HA injection.

The study was carried out at the outpatient clinics of the Department of Physical and Rehabilitative Medicine. Patients were randomly assigned to receive either focused ESWT or HA injections.

The study protocol was approved by the hospital's Ethical Review Board and the study was conducted in accordance with the principles of the Declaration of Helsinki and its amendments. Patients were fully informed of the characteristics of the study before providing consent. This trial has been registered at ClinicalTrials.gov (NCT01783262).

All subjects who received a diagnosis of first CMC joint OA between January 2015 and September 2015, as defined by the guidelines of the American College of Rheumatology, were eligible for the study [20].

A computerized random-number generator was used to formulate the allocation schedule and concealed allocation was carried out with an opaque sealed envelope. Subjects received a diagnosis of first CMC joint OA on the basis of a thorough history, a physical examination, and a radiographic evaluation [20]. Clinical examination included the presence of thumb or wrist pain at rest, tenderness of the trapeziometacarpal joint, joint stiffness, decreased mobility, deformity, instability, and decreased 


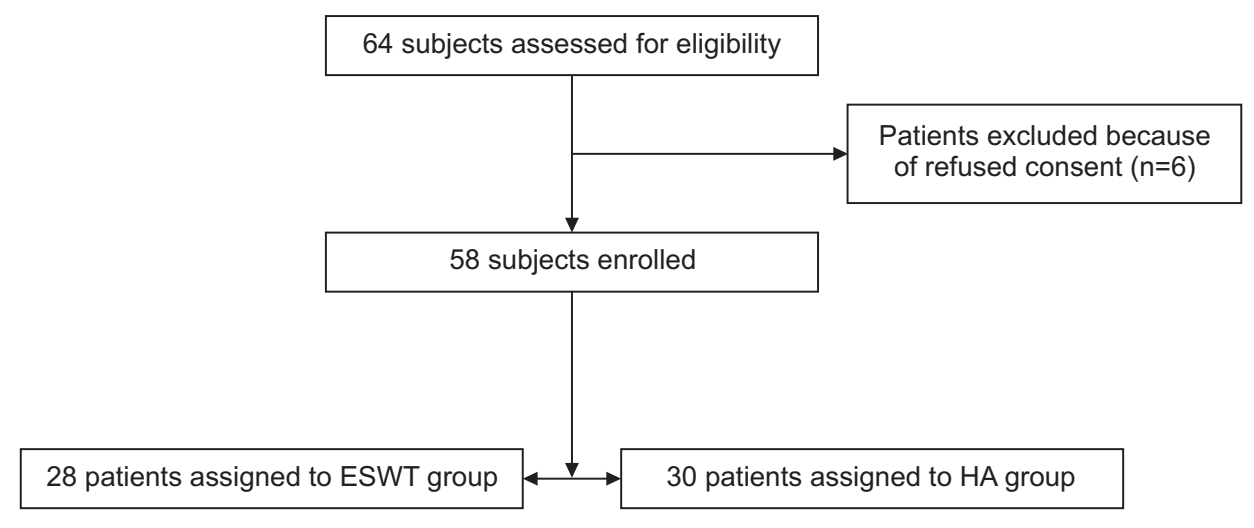

Fig. 1. A flow diagram showing the treatment process and assessment methods. ESWT, extracorporeal shock wave therapy; HA, hyaluronic acid; EFD, energy flux density.

manual function.

A Consolidated Standards of Reporting Trials (CONSORT) diagram for the study is displayed in Fig. 1.

To be eligible for the trial, participants had to have a history of pain at the base of the thumb that had persisted for at least 6 months. Patients did not receive any standard conservative treatment before or during ESWT or HA injection. Other inclusion criteria were (1) subjects over 40 years of age, (2) a minimum score of 4 on the visual analogue scale (VAS), and (3) stage II or III Eaton radiological classification $[21,22]$. The enrolled patients had never received ESWT or injections of HA before.

The main reasons for exclusion from the study were the presence of degenerative or non-degenerative neurologic conditions, a history of hand fractures, coagulopathy, cancer, cardiac pacemaker use, pregnancy, injections of corticosteroids in the hand, and physical therapy in the previous four weeks.

\section{Study intervention}

According to Schmitz et al. [3], the ESWT was performed once a week for 3 consecutive weeks. Moreover, 2,400 consecutive pulses were administered for each treatment session using a frequency of $4 \mathrm{~Hz}$ and an energy flux density (EFD) of $0.09 \mathrm{~mJ} / \mathrm{mm}^{2}$. Focused shock waves were generated electromagnetically with a MODULITH SLK shock wave device (Storz Medical, Tagerwilen, Switzerland) equipped with an in-line ultrasound positioning system.

Patients were seated on a moveable chair and the affected trapeziometacarpal joint was positioned on the treatment head with the forearm supinated to allow for perpendicular application of shock waves with ultrasound guidance on the whole joint from the dorsal to the volar aspects. Ultrasound gel was used as a conductive

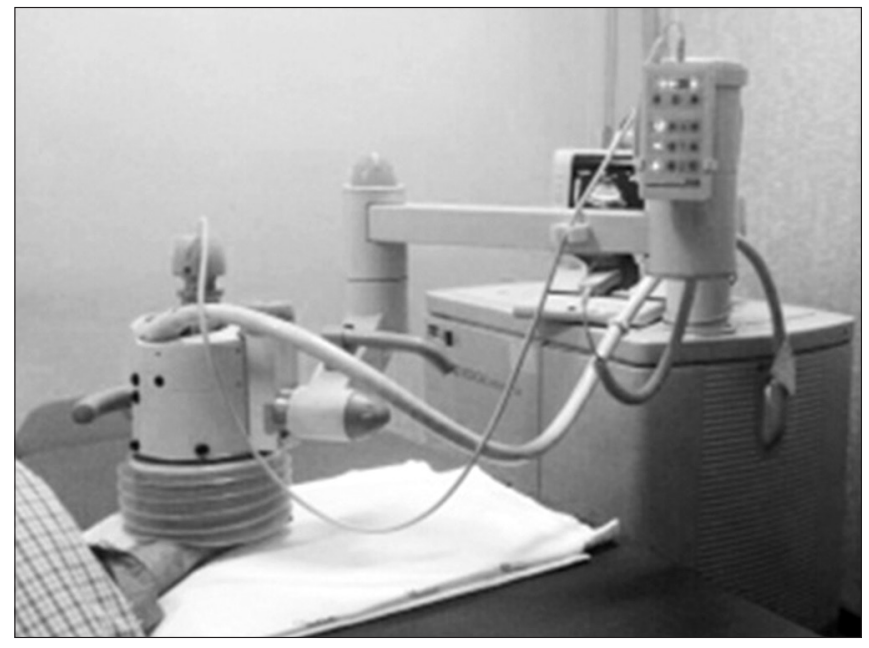

Fig. 2. Extracorporeal shock wave therapy device.

medium between the skin and the treatment head (Fig. 2).

Patients in the HA group underwent one cycle of three injections of $0.5 \mathrm{~cm}^{3} \mathrm{HA}$ (Sinovial Mini) once a week for 3 consecutive weeks. To receive the treatment, patients sat holding their hands in the palm-up position. The first trapeziometacarpal joint space was identified by palpation and the needle tip was inserted lateral to the abductor pollicis longus tendon. The injection was carried out under ultrasound guidance using a 10-MHz probe (Logiq P5 Pro; GE Healthcare, Waukesha, WI, USA) placed transversally for better needle visibility. To avoid bias from different treatment techniques, all ultrasound injections were administered by the same investigator according to the randomization list.

\section{Outcome measures}

Evaluations were carried out at the beginning of treatment (T0), at the end of treatment (T1), and at 3-month (T2) and 6-month (T3) follow-up visits. 


\section{Primary outcome measures}

One of the primary outcomes was an overall reduction of trapeziometacarpal pain, as assessed by a change in mean VAS score $[24,29,30]$ from baseline to follow-up. This scale consists of a $10 \mathrm{~cm}$ horizontal line (with $0 \mathrm{~cm}$ referring to 'no pain' and $10 \mathrm{~cm}$ to the 'the worst pain ever'); patients are asked to rate the intensity of their pain by making a mark on the line. The test-retest reliability is quite good in general, but is higher among literate $(\mathrm{r}=0.94$, $\mathrm{p}<0.001)$ than illiterate patients $(\mathrm{r}=0.71, \mathrm{p}<0.001)$ who were seen before and after attending a rheumatology outpatient clinic [23].

Hand function was measured by changes in the Duruoz Hand Index (DHI) [24] from baseline to follow-up. The index is a self-reported questionnaire that measures hand functional ability by detecting a patient's difficulty in performing eighteen tasks in daily life. Each item is scored from 0 (performed without difficulty) to 5 (impossible to do). A total score is obtained by adding the scores on all questions (range, 0-90). The DHI has been found to be reliable and valid for people with rheumatoid arthritis and osteoarthritis $[24,25]$.

\section{Secondary outcome measures}

The secondary outcomes were average change in grip and pinch strength from baseline to follow-up.

To evaluate grip strength, a hydraulic hand dynamometer was used. Patients were seated with their elbows placed at $90^{\circ}$ of flexion and the wrist was kept in a neutral position between pronation and supination. The dynamometer was set at number 2, in accordance with the instruction manual (PC-5030J1; Preston/Trenton, Ontario, Canada). For each strength test, the scores of three successive trials were recorded and the average value was calculated. Measurements were recorded in kilograms.

A pinch gauge dynamometer was used to evaluate pinch strength. Patients were placed in the same position used for the grip strength test. Three measures were recorded for each pinch (tip and key pinch), for which the mean in kilograms was used for the analysis, in accordance with the instruction manual for the equipment (A853-4; Smith \& Nephew, Germantown, WI, USA). Participants were verbally encouraged to make an effort during all measurements.

\section{Sample size}

A priori power calculation was performed according to the primary outcome measure, assuming a two-tailed $\alpha$ value of 0.05 (sensitivity, 95\%), a $\beta$-value of 0.10 (study power, $90 \%$ ), and an effect size of 0.50 . We determined that at least 25 subjects were required for each group ( $\mathrm{G}^{*}$ Power 3 power analysis program).

\section{Statistical analysis}

Statistical analysis was performed according to the principle of intention-to-treat, with missing data imputed with the 'last observation carried forward' technique. We used parametric tests if data were normally distributed and homogeneous, and non-parametric tests if these two conditions were not satisfied. These assumptions were assessed by Kolmogorov-Smirnov's test and Levene's test, respectively.

The paired t-test was performed to assess differences within each group over time. To evaluate differences between the two groups, the unpaired t-test was performed to compare the mean changes in outcome measures at follow-up vs. baseline. Data are reported as mean \pm standard deviation unless otherwise stated.

The Statistical Package for Social Sciences (SPSS) ver. 18 (SPSS Inc., Chicago, IL, USA) was used for calculations. All data were analyzed by a single researcher. Computed $\mathrm{p}$-values were two-sided, and $\mathrm{p}<0.05$ was taken to indicate statistical significance.

\section{RESULTS}

The groups were homogenous at baseline with regard to gender, age, and disease duration. There were no significant differences in baseline characteristics between the two groups in terms of VAS score, DHI score, or pinch and grip strength. Table 1 reports the baseline characteristics of the study subjects.

Among 58 patients, 28 in the low-energy ESWT group (16 women and 12 men; mean age, $68.03 \pm 9.04$ years; range, 40-80 years) and 30 in the HA group (18 women and 10 men; mean age, $66.67 \pm 8.06$ years; range, $42-78$ years) were treated according to the study protocol.

No side-effects were observed in any of the patients after treatment and the patients themselves did not report any side effects. 


\section{Primary outcome measures}

According to VAS score, a significant change in test performance over time was observed in the ESWT group $(\mathrm{F}=51.45, \mathrm{p}<0.001)$ and in the HA group $(\mathrm{F}=17.96$, $\mathrm{p}<0.001)$.

In the ESWT group, there was a significant decrease in pain between baseline the end of treatment $(p<0.001)$ and between baseline and the visits at the 3rd month $(\mathrm{p}<0.001)$ and 6th month $(\mathrm{p}=0.012)$.

In the HA group, there was a significant decrease in pain between baseline and the end of treatment and be-

Table 1. Baseline characteristics of the study subjects

\begin{tabular}{lccc}
\hline & $\begin{array}{c}\text { ESWT group } \\
(\mathbf{n}=\mathbf{2 8})\end{array}$ & $\begin{array}{c}\text { HA group } \\
(\mathbf{n}=\mathbf{3 0})\end{array}$ & p-value \\
\hline Age (yr) & $68.03 \pm 9.04$ & $66.67 \pm 8.06$ & 0.35 \\
Gender & & & \\
$\quad$ Male & 12 & 10 & 0.55 \\
$\quad$ Female & 16 & 18 & 0.68 \\
$\begin{array}{l}\text { Duration of disease } \\
\text { (yr) }\end{array}$ & $8.2 \pm 3.4$ & $7.9 \pm 4.0$ & 0.22 \\
VAS & $8.01 \pm 1.11$ & $7.62 \pm 1.34$ & 0.34 \\
DHI & $50.89 \pm 13.50$ & $51.56 \pm 14.04$ & 0.22 \\
Finger pinch strength & $4.46 \pm 2.09$ & $4.59 \pm 2.17$ & 0.36 \\
\hline Hand grip strength & $12.87 \pm 3.39$ & $12.46 \pm 3.57$ & 0.45 \\
\hline
\end{tabular}

Values are presented as mean \pm standard deviation or number.

ESWT, extracorporeal shock wave therapy; HA, hyaluronic acid; VAS, visual analogue scale; DHI, Duruoz Hand Index. tween baseline and the 3rd month and 6th month followup visits $(\mathrm{p}<0.001)$. However, a significant increase in pain was found between the visits in the 3rd month and the 6th month ( $\mathrm{p}=0.021$ ) (Fig. 3).

There was a significant difference between groups according to the mean difference between the end of treatment and the 6-month follow-up ( $\mathrm{p}<0.001)$ (Table 2).

According to DHI score, a significant change in DHI test performance over time was observed in the ESWT group $(\mathrm{F}=18.82, \mathrm{p}<0.001)$ and the HA group $(\mathrm{F}=67.35, \mathrm{p}<0.001)$. Both groups experienced significant improvements in hand function between baseline and the end of treatment $(\mathrm{p}<0.001)$ and between baseline and 3 months $(\mathrm{p}<0.001)$ and 6 months $(\mathrm{p}=0.012)$. There were no significant differences between the two groups in terms of improvement

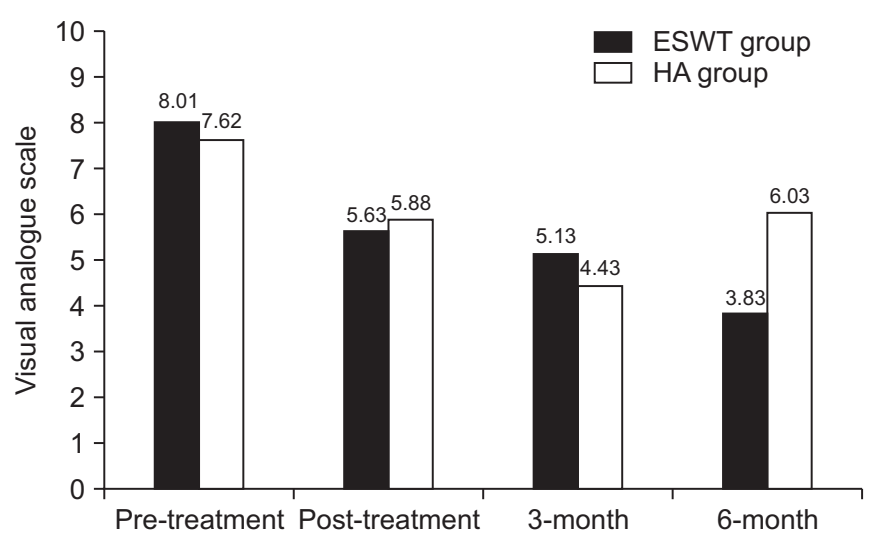

Fig. 3. Changes over time of visual analogue scale score in both groups. ESWT, extracorporeal shock wave therapy; HA, hyaluronic acid.

Table 2. Differences between the ESWT and HA groups in primary outcome measures

\begin{tabular}{|c|c|c|c|c|}
\hline & \multicolumn{4}{|c|}{ ESWT group vs. HA group } \\
\hline & \multirow{2}{*}{ Mean difference } & \multicolumn{2}{|c|}{ 95\% CI } & \multirow{2}{*}{ p-value } \\
\hline & & Low & Upper & \\
\hline \multicolumn{5}{|l|}{ VAS } \\
\hline T1 vs. T0 & -0.17 & -1.34 & 0.99 & 0.76 \\
\hline T2 vs. T0 & 0.07 & -1.02 & 1.16 & 0.89 \\
\hline T3 vs. T0 & -2.60 & -3.68 & -1.52 & $<0.001$ \\
\hline \multicolumn{5}{|l|}{ DHI } \\
\hline T1 vs. T0 & -1.63 & -11.32 & 8.0 & 0.73 \\
\hline T2 vs. T0 & 0.49 & -7.69 & 8.68 & 0.90 \\
\hline T3 vs. T0 & -3.66 & -8.08 & 6.61 & 0.80 \\
\hline
\end{tabular}

ESWT, extracorporeal shock wave therapy; HA, hyaluronic acid; CI, confidence interval; VAS, visual analogue scale; DHI, Duruoz Hand Index; T0, baseline value; T1, end of treatment value; T2, 3-month follow-up value; T3, 6-month follow-up. 
of DHI score at the time of the follow-up visits.

\section{Secondary outcome measures}

When we tested finger pinch strength, we found that the ESWT group showed a significant improvement from the end of treatment $(\mathrm{p}=0.03)$ to the 6 -month followup $(\mathrm{p}=0.002)$, whereas in the HA group there was a significant change in score only at the 6 -month follow-up $(p=0.017)$ (Table 3). There were no significant differences between the two groups (Table 4).

In terms of hand grip strength, the ESWT group showed significant improvement at the 6-month follow-up $(\mathrm{p}<0.001)$, while in the HA group there was a significant change in score up to the 6 month follow-up ( $\mathrm{p}=0.002)$ (Table 3). There were no significant differences between the two groups (Table 4).

\section{DISCUSSION}

First CMC OA can involve a high degree of pain and functional disability, making it difficult for sufferers to perform daily activities [9]. Management of the disease is controversial and there are only a few high-quality studies addressing the efficacy of conservative treatments [35].

Among the available therapies, the best results seem to be produced by intra-articular injection of corticosteroids or HA in terms of pain relief and improvement in hand function. In particular, intra-articular corticosteroid use appears to be more efficient in relieving acute pain, while intra-articular HA has a more gradual but prolonged effect over time $[8,26,27]$.

In recent years, ESWT has shown significant clinical ef-

Table 3. Differences between the ESWT group and HA group in secondary outcome measures

\begin{tabular}{|c|c|c|c|c|}
\hline & ESWT group & p-value (vs. T0) & HA group & p-value (vs. T0) \\
\hline \multicolumn{5}{|c|}{ Finger pinch strength (kgf) } \\
\hline $\mathrm{T} 1$ & $6.14 \pm 1.23$ & 0.030 & $5.15 \pm 1.87$ & 0.050 \\
\hline $\mathrm{T} 2$ & $4.94 \pm 1.91$ & 0.440 & $5.34 \pm 2.06$ & 0.150 \\
\hline T3 & $5.86 \pm 1.81$ & 0.002 & $5.97 \pm 1.75$ & $<0.017$ \\
\hline \multicolumn{5}{|c|}{ Hand grip strength (kgf) } \\
\hline $\mathrm{T} 1$ & $13.33 \pm 3.13$ & 0.054 & $14.79 \pm 4.28$ & 0.003 \\
\hline $\mathrm{T} 2$ & $15.68 \pm 3.94$ & 0.001 & $16.73 \pm 4.67$ & $<0.001$ \\
\hline T3 & $16.86 \pm 4.75$ & $<0.001$ & $15.64 \pm 4.93$ & 0.002 \\
\hline
\end{tabular}

Values are presented as mean \pm standard deviation.

ESWT, extracorporeal shockwave therapy; HA, hyaluronic acid; T0, baseline value; T1, end of treatment value; T2, 3-month follow-up value; T3, 6-month follow-up.

Table 4. Differences between the ESWT group and the HA group according to grip and pinch strength

\begin{tabular}{|c|c|c|c|c|}
\hline & \multicolumn{4}{|c|}{ ESWT groups vs. HA group } \\
\hline & \multirow{2}{*}{ Mean difference } & \multicolumn{2}{|c|}{ 95\% CI } & \multirow{2}{*}{ p-value } \\
\hline & & Low & Upper & \\
\hline \multicolumn{5}{|c|}{ Grip strength } \\
\hline T1 vs. T0 & -1.88 & -3.42 & -0.33 & 0.18 \\
\hline T2 vs. T0 & -1.46 & -3.82 & 0.91 & 0.22 \\
\hline T3 vs. T0 & 0.79 & -1.78 & 3.38 & 0.54 \\
\hline \multicolumn{5}{|c|}{ Pinch strength } \\
\hline T1 vs. T0 & 1.11 & -0.02 & 2.25 & 0.05 \\
\hline T2 vs. T0 & -0.27 & -1.85 & 1.30 & 0.73 \\
\hline T3 vs. T0 & 0.15 & -1.36 & 1.39 & 0.98 \\
\hline
\end{tabular}

ESWT, extracorporeal shock wave therapy; HA, hyaluronic acid; CI, confidence interval; T0, baseline value; T1, end of treatment value; T2, 3-month follow-up value; T3, 6-month follow-up. 
fects in a wide range of musculoskeletal disorders [28-30] owing to its angiogenic analgesic and anti-inflammatory effects $[14,15,31,32]$.

We surmised that ESWT could have a beneficial effect on reduction of pain and improvements in hand function even in patients suffering from OA. Nowadays, indeed, $\mathrm{OA}$ is not considered a non-inflammatory disorder as it has been shown to be associated with several inflammatory mediators [33].

The results of our study showed that individuals in both the ESWT group and the HA group improved significantly at the 6-month follow-up visit in terms of pain and hand function. We observed a higher average improvement in painful symptomatology at the 6-month follow-up visit in the ESWT group compared to the HA group. Furthermore, according to the results of the pinch and grip tests, we found a significant improvement in strength in both groups, but the ESWT group showed superior improvement on the pinch test beginning immediately at the end of treatment while in the HA group the improvement started at the 6-month follow-up.

Basic science research has shown that ESWT use for osteoarthritis is safe with proper dosing [34]. However, discussion is still ongoing as to which combination of parameters would be the most useful to maximize the efficacy of ESWT treatment for a specific indication [35]. The number of required treatment sessions and the energy level used are two relevant parameters that should be considered. The need for standardization of treatment regimens via randomized controlled trials established one to three ESWT sessions at weekly intervals as standard clinical practice regardless of underlying pathology. A systematic review recommended the use of three treatment sessions at 1-week intervals, with 2,000 impulses per session and the highest EFD the patient can tolerate [36].

Therefore, we administered 2,400 consecutive pulses during each weekly treatment session for 3 consecutive weeks at an EFD of $0.09 \mathrm{~mJ} / \mathrm{mm}^{2}$.

The results of our study can be explained by taking into account the analgesic and anti-inflammatory effects of ESWT, which are characterized by down-regulation of $\mathrm{TNF} \alpha, \mathrm{IL}-10, \mathrm{NF}-\mathrm{kB}$, and the levels of NF-kB-dependent genes produced by chondrocytes $[15,18]$.

Moreover, ESWT causes an increase in nitric oxide (NO) and vascular endothelial growth factor (VEGF) and a re- duction of leukocyte and macrophage infiltration, which may lead to a reduction in the inflammatory process [37]. So far, only a few studies have evaluated the effectiveness of ESWT in the management of OA in animal models.

Dahlberg et al. [16] applied EWST in dogs with persistent stifle lameness and found a trend toward increased $\mathrm{ROM}(\mathrm{p}=0.07)$ and a positive slope in the treatment group compared to the control group. In an experimental rabbit model, Zhao et al. [38] observed that ESWT may change the activity of OA by reducing chondrocyte apoptosis.

Our findings are in accordance with the results of previous studies, which demonstrated the efficacy of ESWT in patients suffering from knee osteoarthritis [19,39].

Zhao et al. [39] demonstrated the effectiveness of ESWT compared with placebo in reducing pain and improving knee function in patients with knee $\mathrm{OA}$ at a 12 -week follow-up.

In a more recent study, Kim et al. [19] divided 60 patients into two groups: a low-energy group (EFD, 0.040 $\mathrm{mJ} / \mathrm{mm}^{2}$ ) and a medium-energy group (EFD, $0.093 \mathrm{~mJ} /$ $\mathrm{mm}^{2}$ ). The latter showed a greater improvement in pain relief and knee function than the former.

The results of our study provide further evidence that HA may be a useful conservative treatment in the management of first CMC OA even though it is an invasive procedure which can be painful and may put the patient at a risk of infection [33].

Shock waves are a promising treatment option because they are non-invasive and they seem to be an equivalent and possibly better alternative to HA injection in the treatment of first CMC joint OA, particularly in patients with moderate-to-severe pain.

The present study has some limitations, namely the absence of a placebo group and a relatively small number of patients.

We conclude that the use of ESWT in patients with CMC first OA leads to a reduction in pain, an improvement in pinch test performance at the 6-month follow-up, and a decrease in hand disability up to the 6-month follow-up. The pain reduction effect of ESWT is likely to be superior to that of HA injection immediately and after 6 months. In terms of improvements in hand function and strength, ESWT appears to be equal to HA.

Therefore, the results are encouraging and it would be interesting to carry out further studies on the long-term 
effects of this technique on a larger number of patients.

\section{CONFLICT OF INTEREST}

No potential conflict of interest relevant to this article was reported.

\section{REFERENCES}

1. Arden NK, Leyland KM. Osteoarthritis year 2013 in review: clinical. Osteoarthritis Cartilage 2013;21:140913.

2. Wajon A, Vinycomb T, Carr E, Edmunds I, Ada L. Surgery for thumb (trapeziometacarpal joint) osteoarthritis. Cochrane Database Syst Rev 2015;2:CD004631.

3. Heyworth BE, Lee JH, Kim PD, Lipton CB, Strauch RJ, Rosenwasser MP. Hylan versus corticosteroid versus placebo for treatment of basal joint arthritis: a prospective, randomized, double-blinded clinical trial. J Hand Surg Am 2008;33:40-8.

4. Cook GS, Lalonde DH. MOC-PSSM CME article: management of thumb carpometacarpal joint arthritis. Plast Reconstr Surg 2008;121:1-9.

5. Davis AM, MacKay C. Osteoarthritis year in review: outcome of rehabilitation. Osteoarthritis Cartilage 2013;21:1414-24.

6. Mader R, Lavi I, Luboshitzky R. Evaluation of the pituitary-adrenal axis function following single intraarticular injection of methylprednisolone. Arthritis Rheum 2005;52:924-8.

7. Gigante A, Callegari L. The role of intra-articular hyaluronan (Sinovial) in the treatment of osteoarthritis. Rheumatol Int 2011;31:427-44.

8. Spaans AJ, van Minnen LP, Kon M, Schuurman AH, Schreuders AR, Vermeulen GM. Conservative treatment of thumb base osteoarthritis: a systematic review. J Hand Surg Am 2015;40:16-21.

9. Monfort J, Rotes-Sala D, Segales N, Montanes FJ, Orellana C, Llorente-Onaindia J, et al. Comparative efficacy of intra-articular hyaluronic acid and corticoid injections in osteoarthritis of the first carpometacarpal joint: results of a 6-month single-masked randomized study. Joint Bone Spine 2015;82:116-21.

10. Ioppolo F, Tattoli M, Di Sante L, Attanasi C, Venditto T, Servidio M, et al. Extracorporeal shock-wave therapy for supraspinatus calcifying tendinitis: a randomized clinical trial comparing two different energy levels. Phys Ther 2012;92:1376-85.

11. Peled E, Portal-Banker T, Norman D, Melamed E. Plantar fasciitis and extracorporeal shock wave therapy: essence, diagnosis and treatment methods. Harefuah 2011;150:122-6.

12. Sems A, Dimeff R, Iannotti JP. Extracorporeal shock wave therapy in the treatment of chronic tendinopathies. J Am Acad Orthop Surg 2006;14:195-204.

13. Ioppolo F, Rompe JD, Furia JP, Cacchio A. Clinical application of shock wave therapy (SWT) in musculoskeletal disorders. Eur J Phys Rehabil Med 2014;50: 217-30.

14. Wang CJ, Huang HY, Pai CH. Shock wave-enhanced neovascularization at the tendon-bone junction: an experiment in dogs. J Foot Ankle Surg 2002;41:16-22.

15. Mariotto S, de Prati AC, Cavalieri E, Amelio E, Marlinghaus E, Suzuki H. Extracorporeal shock wave therapy in inflammatory diseases: molecular mechanism that triggers anti-inflammatory action. Curr Med Chem 2009;16:2366-72.

16. Dahlberg J, Fitch G, Evans RB, McClure SR, Conzemius $\mathrm{M}$. The evaluation of extracorporeal shockwave therapy in naturally occurring osteoarthritis of the stifle joint in dogs. Vet Comp Orthop Traumatol 2005;18: 147-52.

17. Revenaugh MS. Extracorporeal shock wave therapy for treatment of osteoarthritis in the horse: clinical applications. Vet Clin North Am Equine Pract 2005;21:60925.

18. Moretti B, Iannone F, Notarnicola A, Lapadula G, Moretti L, Patella V, et al. Extracorporeal shock waves down-regulate the expression of interleukin-10 and tumor necrosis factor-alpha in osteoarthritic chondrocytes. BMC Musculoskelet Disord 2008;9:16.

19. Kim JH, Kim JY, Choi CM, Lee JK, Kee HS, Jung KI, et al. The dose-related effects of extracorporeal shock wave therapy for knee osteoarthritis. Ann Rehabil Med 2015;39:616-23.

20. Altman R, Alarcon G, Appelrouth D, Bloch D, Borenstein D, Brandt $\mathrm{K}$, et al. The American College of Rheumatology criteria for the classification and reporting of osteoarthritis of the hand. Arthritis Rheum 1990;33:1601-10.

21. Eaton RG, Littler JW. Ligament reconstruction for the painful thumb carpometacarpal joint. J Bone Joint 
Surg Am 1973;55:1655-66.

22. Hirschfeld M, Galan A, Arenas J, Del Aguila B, BenitezParejo N, Costa JA, et al. Inter-observer agreement on the Eaton-Littler classification of trapeziometacarpal joint osteoarthritis. Rev Esp Cir Ortop Traumatol 2014; 58:237-41.

23. Hawker GA, Mian S, Kendzerska T, French M. Measures of adult pain: visual analog scale for pain (VAS Pain), numeric rating scale for pain (NRS Pain), mcgill pain questionnaire (MPQ), short-form mcgill pain questionnaire (SF-MPQ), chronic pain grade scale (CPGS), short form-36 bodily pain scale (SF-36 BPS), and measure of intermittent and constant osteoarthritis pain (ICOAP). Arthritis Care Res (Hoboken) 2011;63 Suppl 11:S240-52.

24. Duruoz MT, Poiraudeau S, Fermanian J, Menkes CJ, Amor B, Dougados M, et al. Development and validation of a rheumatoid hand functional disability scale that assesses functional handicap. J Rheumatol 1996; 23:1167-72.

25. Brower LM, Poole JL. Reliability and validity of the Duruoz Hand Index in persons with systemic sclerosis (scleroderma). Arthritis Rheum 2004;51:805-9.

26. Fuchs S, Monikes R, Wohlmeiner A, Heyse T. Intra-articular hyaluronic acid compared with corticoid injections for the treatment of rhizarthrosis. Osteoarthritis Cartilage 2006;14:82-8.

27. Stahl S, Karsh-Zafrir I, Ratzon N, Rosenberg N. Comparison of intraarticular injection of depot corticosteroid and hyaluronic acid for treatment of degenerative trapeziometacarpal joints. J Clin Rheumatol 2005;11: 299-302.

28. Notarnicola A, Moretti B. The biological effects of extracorporeal shock wave therapy (ESWT) on tendon tissue. Muscles Ligaments Tendons J 2012;2:33-7.

29. Notarnicola A, Maccagnano G, Tafuri S, Fiore A, Margiotta C, Pesce V, et al. Prognostic factors of extracorporeal shock wave therapy for tendinopathies. Musculoskelet Surg 2016;100:53-61.

30. Maffulli G, Hemmings S, Maffulli N. Assessment of the effectiveness of extracorporeal shock wave therapy (eswt) for soft tissue injuries (ASSERT): an online database protocol. Transl Med UniSa 2014;10:46-51.

31. Wang CJ, Wang FS, Yang KD, Weng LH, Hsu CC, Huang CS, et al. Shock wave therapy induces neovascularization at the tendon-bone junction: a study in rabbits. J Orthop Res 2003;21:984-9.

32. Ochiai N, Ohtori S, Sasho T, Nakagawa K, Takahashi K, Takahashi N, et al. Extracorporeal shock wave therapy improves motor dysfunction and pain originating from knee osteoarthritis in rats. Osteoarthritis Cartilage 2007;15:1093-6.

33. Iannitti T, Lodi D, Palmieri B. Intra-articular injections for the treatment of osteoarthritis: focus on the clinical use of hyaluronic acid. Drugs R D 2011;11:1327.

34. Renz H, Rupp S. Effects of shock waves on chondrocytes and their relevance in clinical practice. Arch Orthop Trauma Surg 2009;129:641-7.

35. Lohrer H, Nauck T, Korakakis V, Malliaropoulos N. Historical ESWT Paradigms are overcome: a narrative review. Biomed Res Int 2016;2016:3850461.

36. Schmitz C, Csaszar NB, Milz S, Schieker M, Maffulli $\mathrm{N}$, Rompe JD, et al. Efficacy and safety of extracorporeal shock wave therapy for orthopedic conditions: a systematic review on studies listed in the PEDro database. Br Med Bull 2015;116:115-38.

37. Mittermayr R, Antonic V, Hartinger J, Kaufmann H, Redl H, Teot L, et al. Extracorporeal shock wave therapy (ESWT) for wound healing: technology, mechanisms, and clinical efficacy. Wound Repair Regen 2012;20:456-65.

38. Zhao Z, Ji H, Jing R, Liu C, Wang M, Zhai L, et al. Extracorporeal shock-wave therapy reduces progression of knee osteoarthritis in rabbits by reducing nitric oxide level and chondrocyte apoptosis. Arch Orthop Trauma Surg 2012;132:1547-53.

39. Zhao Z, Jing R, Shi Z, Zhao B, Ai Q, Xing G. Efficacy of extracorporeal shockwave therapy for knee osteoarthritis: a randomized controlled trial. J Surg Res 2013; 185:661-6. 